\title{
Expression Responses of the Complement Components in Zebrafish Organs after Stimulation with Poly I:C, Mimicry of Viral Infection
}

Vo, Kha Tam

Laboratory of Marine Biochemistry, Division of Marine Biological Chemistry, Department of Bioscience and Biotechnology, Graduate School of Bioresource and Bioenvironmental Science, Kyushu University

Tsujikura, Masakazu

Laboratory of Marine Biochemistry, Division of Marine Biological Chemistry, Department of Bioscience and Biotechnology, Graduate School of Bioresource and Bioenvironmental Science, Kyushu University

Somamoto, Tomonor i

Laboratory of Marine Biochemistry, Division of Marine Biological Chemistry, Department of Bioscience and Biotechnology, Faculty of Agriculture, Kyushu University

Nakano, Miki

Laboratory of Marine Biochemistry, Division of Marine Biological Chemistry, Department of Bioscience and Biotechnology, Faculty of Agriculture, Kyushu University

https://doi.org/10.5109/16120

出版情報：九州大学大学院農学研究院紀要. 54 (2)，pp. 389-395，2009-10-29. Faculty of Agriculture, Kyushu University

バージョン :

権利関係: 


\title{
Expression Responses of the Complement Components in Zebrafish Organs after Stimulation with Poly I:C, Mimicry of Viral Infection
}

\author{
VO Kha Tam¹, Masakazu TSUJIKURA ${ }^{1}$, Tomonori SOMAMOTO \\ and Miki NAKAO*
}

\author{
Laboratory of Marine Biochemistry, Division of Marine Biological Chemistry, \\ Department of Bioscience and Biotechnology, Faculty of Agriculture, \\ Kyushu University, Hakozaki, Fukuoka 812-8581, Japan \\ (Received June 26, 2009 and accepted July 13, 2009)
}

\begin{abstract}
Defensive roles of the complement system in fish are largely unknown, although expressions of several complement components have been examined in some teleost species upon bacterial and parasitic infections. To infer possible anti-virus defensive roles of the complement system in teleost, zebrafish were simulated by intraperitoneal injection of poly I: C, a double strand RNA analogue, to mimic viral infection, and expression of 24 complement components and their subunits in the gill, brain, kidney, gut, hepatopancreas, gonad, heart, spleen, and skin were analyzed by reverse-transcription PCR. In normal (unstimulated) fish, the hepatopancreas showed highest expression of most genes tested, and considerable extra hepatic expression was also observed for many complement components. Upon stimulation, the hepatopancreas showed decreased expression of CD11/CD18, B/C2-A3, MASP3, and properdin, while marked up-regulation of several complement component genes was observed in the gill, suggesting an important but unknown role of the gill in host defense against viral infection
\end{abstract}

\section{INTRODUCTION}

Gene expression patens such as tissue distribution and response to any stimuli at the mRNA level provide important information to infer the gene function. As the complement encoding genes have increasingly been clarified, a number of approach to elucidate their functions in host defense and other biological processes have been performed for a few fish species in which cDNA or genomic sequence of the complement components are available. For example, expression responses of various complement components have been analyzed for several fish species against infection of bacteria (Raida and Buchmann, 2009; Gerwick et al., 2007; Peatman et al., 2007; Overturf and LaPatra, 2006) and parasites (Gonzalez et al., 2007a; Gonzalez et al., 2007b; Saeij et al., 2003; Sigh et al., 2004; Alvarez-Pellitero, 2008), and after vaccination (Domrongphol et al., 2009; von Gersdorff Joegensen et al., 2008; Park et al., 2005) and immunostimulant administration (Selvaraj et al., 2006; Selvaraj et al., 2005; Lovoll et al., 2007). However, much less is known about expression response of complement components after viral infection of fish. In the present study, the expression of the complement component genes identified in the accompanying paper (Vo et al., 2009) and MBL were examined for a wide range of organs of zebrafish after administration of poly I:C, a double strand RNA analogue that mimics viral infection (Fortier et al., 2004).

1 Laboratory of Marine Biochemistry, Division of Marine Biological Chemistry, Department of Bioscience and Biotechnology, Graduate School of Bioresource and Bioenvironmental Science, Kyushu University

* Corresponding author (E-mail: mikimnakao@kyudai.jp)

\section{MATERIALS AND METHODS}

\section{Fish}

Adult zebrafish (Danio rerio), weighing about $1 \mathrm{~g}$, were purchased from a local pet shop and maintained in a circulating tank at $26^{\circ} \mathrm{C}$ and acclimated at least two weeks before use with daily feeding with commercial pellet.

\section{Reagents}

Polyinosine-polycytidylic acid sodium salt (Poly I:C), obtained from Sigma (P 0913, USA), was dissolved in $10 \mathrm{mM}$ phosphate-buffered saline (pH 7.5) at a concentration of $10 \mathrm{mg} / \mathrm{ml}$ and stored at $-20{ }^{\circ} \mathrm{C}$ until use. Oligonucleotide primers were synthesized and supplied from GeneNet (Fukuoka, Japan). KOD Plus DNA polymerase was obtained from Toyobo (Tokyo, Japan).

\section{Stimulation of fish and isolation of RNA}

Poly I:C in PBS was intraperitoneally injected to zebrafish at a dose of $10 \mu \mathrm{g} / \mathrm{g}$-body weight. PBS was injected to control fish. One day later, fish were anesthetized in $400 \mathrm{ppm}$ quinaldine, and sacrificed for collection of the gills, brain, kidney, gut, hepatopancreas, gonad, heart, spleen, and skin (10-20 mg). The organs were rapidly homogenized in $200 \mu \mathrm{l}$ of ISOGEN, and total RNA was isolated according to the manufacturer's instructions and stored at $-80^{\circ} \mathrm{C}$.

\section{RT-PCR}

First strand cDNA was synthesized from the total RNA (1 $\mu \mathrm{g}$ ) using MMLV-reverse transcriptase and oligo (dT) primer following the protocol provided with the enzyme. The cDNA equivalent to about $0.025 \mu \mathrm{g}$ RNA was used as a template of PCR reaction with KOD Plus DNA polymerase kit (Toyobo, Japan). Primer sequences used for each complement component gene and target 
sizes of amp icons are listed in Table 1. Beta-actin mRNA was amplified as a positive control.

PCR amplification was conducted on a T3 thermal cycler (Biometra, Germany) under the following conditions: $94^{\circ} \mathrm{C}$ for $15 \mathrm{sec}, 53{ }^{\circ} \mathrm{C}-57^{\circ} \mathrm{C}$ for $15 \mathrm{sec}, 68^{\circ} \mathrm{C} 1 \mathrm{~min}$, 30 cycles. The annealing temperature for each primer set is shown in Table 1. The amplified products were run on $2 \%$ agarose gels in Tris-Borate-EDTA buffer $(\mathrm{pH} 8.2)$ containing $0.5 \mu \mathrm{l} / \mathrm{ml}$ ethidium bromide (EtBr).

\section{RESULTS}

Expression patterns at the mRNA level of all the complement component genes identified the accompanying paper (Vo et al., 2009) and the mannose-binding lectin (Nakao et al., 2006) were evaluated by RT-PCR, using three fish each for control and test groups. Only the results of two representative fish are shown in the figures of the following sections. Authenticity of the amplicon was confirmed by direct sequencing for each gene (data not shown).

\section{Expression pattern in the gills}

In the gill of control fish (Fig. 1), mRNA of the following genes were detected as a significant band: C1qA, B, C-chains, C1r/s, C3-1, C4-2, C6, CR1, CD11/CD18, B/ $\mathrm{C} 2-\mathrm{A} 3$, and $\mathrm{P}$, although the expressions of CR1, B/C2-A3, and $\mathrm{P}$ were obvious only in one of two individuals. Signals
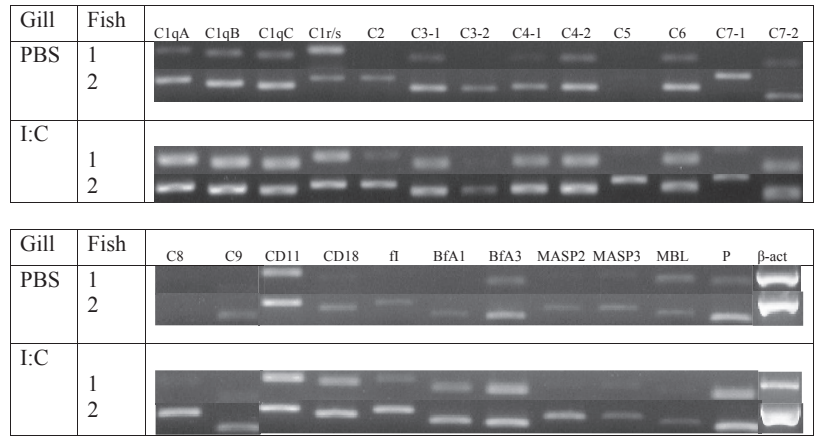

Fig. 1. Expression of the complement component genes in the gills. After RT-PCR amplification, the PCR products were run on a agarose gel containing $0.5 \mu \mathrm{g} / \mathrm{ml}$ EtBr. Results from two fish (number 1 and 2 ) are shown. PBS denotes the control fish group injected with PBS alone, and I:C stands for the stimulated fish group administered with poly I:C. Sizes of the amplicons are shown in Table 1. Abbreviations: C2, B/ $\mathrm{C} 2-\mathrm{B}$; fI, factor I; BfA1, B/C2-A1; BfA3, B/C2-A3; MBL, mannose-binding lectin; MASP, MBL-associated serine protease; $\mathrm{P}$, properdin; $\beta$-act, $\beta$-actin.

of B/C2-B, C3-2, C4-1, C5, C7-1, C7-2, C8, C9, fI, MASP2, MASP3, and MBL were only faint or not detected in the normal gills. In the poly I:C-injected fish, amplicon bands of all the genes except for MASP3 and MBL became visible at least in one of the two test fish, suggesting that the

Table 1. RT-PCR primers and expected cDNA amplicon size

\begin{tabular}{|c|c|c|}
\hline $\begin{array}{l}\text { Gene/ } \\
\text { Annealing } \\
\text { temperature }\left({ }^{\circ} \mathrm{C}\right) / \\
\text { Amplicon length }(\mathrm{bp})\end{array}$ & $\begin{array}{c}\text { Sense primer } \\
\text { sequence (5'-3') }\end{array}$ & $\begin{array}{l}\text { Anti-sense primer } \\
\text { sequence }\left(5^{\prime}-3^{\prime}\right)\end{array}$ \\
\hline $\mathrm{C} 1 \mathrm{qA} / 57 / 138$ & TTATGTCTCAGACTTAAGAGCACAAGTGCG & ATTGAACACTGCATACAAGCGTTTGGGCAT \\
\hline $\mathrm{C} 1 \mathrm{qB} / 57 / 118$ & AACAGTCTGCCTGTCTAAAGATTCAGGTAG & GTGCAGTCAGTGTCTCTACTAAACACAGTA \\
\hline $\mathrm{C} 1 \mathrm{qC} / 55 / 106$ & AAGCTGTGTGTTATCCTAGTGCATGATGAT & CTGTTGCATACATGCCATTAAGGGCATTTG \\
\hline $\mathrm{C} 1 \mathrm{r} / \mathrm{s} / 55 / 138$ & AACAGGTGAAGCTGTTAAAGAGGGCATGAT & CCTCTACAACTGTCAATACCATGAACATCA \\
\hline $\mathrm{C} 2 / 55 / 181$ & TATGATTACGACATCGCTCTGATCAGGAT & ATGTGTTCCCTGTGAGATGAAGTGA \\
\hline C3-1/57/106 & CGCCTGGCAGCAATGTCCAG & GATTGAAGACTTCACACCTTTCTCTGCCTT \\
\hline C3-2/55/106 & CGCCTGGCAGCAATGTTCAT & GATCGAAGATTTCACACCTTTTTCTGCAAA \\
\hline C4-1/57/104 & TAATCACTGATGTTCTGCAGACTGGTAGGA & GTTATTCTGCTCTGAGATTGCTCTGTCATG \\
\hline $\mathrm{C} 4-2 / 57 / 100$ & CACCAAAGACTCAAGTGGAAACATGCA & CAGATTGCATCCATCAACTTGGTACTCTTT \\
\hline $\mathrm{C} 5 / 55 / 151$ & GGAAGATCTAGATGAGTACCAGAATGGACT & TGTGCACTTAAATGCTGGAGCTTGATATTC \\
\hline $\mathrm{C} 6 / 55 / 105$ & TCAGTGTCCAAAACGTGTGAGTGTAAGATG & TTCATGCTGAAGCTCAAACTCTATACTGCT \\
\hline $\mathrm{C} 7-1 / 57 / 155$ & ACTGCTGGAGCTGGACGCAAATTATATGAT & CCAGCGATATAAGCGCTATTTCCTCCTATA \\
\hline $\mathrm{C} 7-2 / 55 / 82$ & GTACCTCACAGAGAAACACTCATGTC & TCCCATATTCGCAGGCTTGG \\
\hline $\mathrm{C} 8 / 57 / 172$ & CCTATAATGACCACAAATACAAGCGATCGG & TTGATGAAATGAGTGCCGTAGTCTTGGTAG \\
\hline C9/57101 & GTATCGCTCTAGGAGTATTGAAGTCTTTGG & CTTGTAAAGGTGCACTGCCACTGAG \\
\hline CD11/55/143 & CCTCATAAATCACAGATCACAGTGAAG & GTCTTTAGCATCTGTGTCCATGAGA \\
\hline CD18/57/156 & CTCTACTCTGTCAGTGTCCTTAAAGACAGA & CTGGAATAATGGGTTGGTACCAGAAGTCTT \\
\hline $\mathrm{fI} / 57 / 178$ & AAGGTGACGATTATTGGTGACTGCCAGAAT & AGTCGAAGTAATGTGCCACTTTGGTGTAGA \\
\hline BfA1/55/131 & TGTGCAGTGGTGGTAATCAACCACA & TATGGTAATCTCTGGAGTCTGAAACCGAAA \\
\hline BfA3/55/127 & AGCGGTGGTATTGACCCCAC & ATGGTAATCTCTGGTGTAAGACTCAGATCT \\
\hline MASP2/55/152 & ACGGAGAATATGATTTGTGCTGGGTTTTCC & GACATGACTTTTCAATCCATGAAAGGTAG \\
\hline MASP3/55/155 & TTAACCCCTAACACTTTGGGTATTGTAGCC & AAAGCCAGCGCAGAACATATTACCAGTGAT \\
\hline MBL/57/129 & ATGTTGGTGCAACAGATGCAAAGAAAGAGG & GTTCACACACCACATGCCACTTTGAATTAC \\
\hline $\mathrm{P} / 57 / 107$ & TGGAGAAATTGTTGAACATGGCAGCTGCTT & CGGATTGGTATTCAGAAATGTCAGGAACAC \\
\hline$\beta-$ act $/ 53 / 1160$ & GTTGACAACGGCTCCGGTAT & CAGAAGCCATGCTGATGTCA \\
\hline
\end{tabular}

Abbreviations: C2, B/C2-B; BfA1, B/C2-A1; BfA3, B/C2-A3; fI, factor I; MBL, mannose-binding lectin; MASP, MBLassociated serine protease; $\mathrm{P}$, properdin; $\beta$-act, $\beta$-actin. 
expression of these genes were up-regulated in the gills by poly I:C stimulation. Especially, up-regulation of B/ C2-B, C4-1 and B/C2-A1 seemed prominent.

\section{Expression pattern in the brain}

Brain of the control fish showed expression of a wide range of complement components: C1qA, B, C-chains, C1r/s, C3-1, C4-1, C4-2, C6, C7-1, C7-2, C8, CR1, CD11/ CD18, fI, B/C2-A3, MASP3, MBL, and P, whereas only a faint signal was observed for $\mathrm{B} / \mathrm{C} 2-\mathrm{B}, \mathrm{B} / \mathrm{C} 2-\mathrm{A} 1$, and MASP2, and no detectable band was obtained for C3-2, C5, and C9 (Fig. 2). In the stimulated fish, on the other hand, many genes gave apparently stronger signals at least in one of the two injected fish, whilst expression levels of C1qA, B, C-chains, C3-1, C7-2, CR1, and CD11/ CD18 were kept flat.
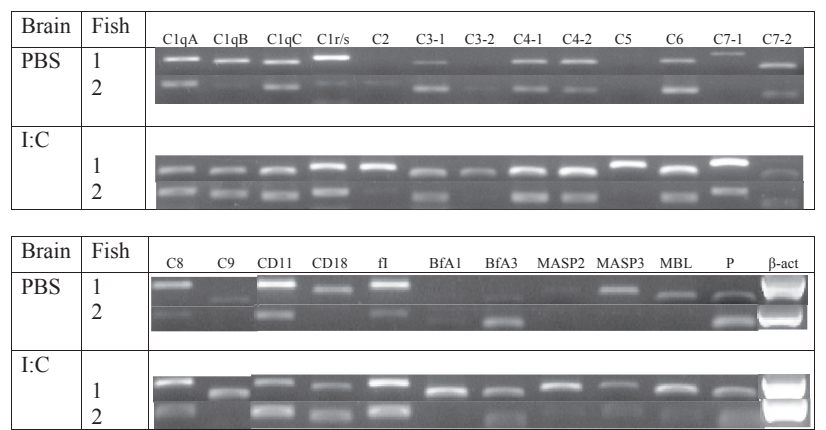

Fig. 2. Expression of the complement component genes in the brains. Abbreviations are shown in the Fig. 1 captions.

\section{Expression pattern in the kidney}

In the kidney, at least one of the two control fish gave significant signals of expression of all the genes tested, and no obvious up-regulation was recognized for any of the genes in the poly I:C-stimulated fish (Fig. 3).
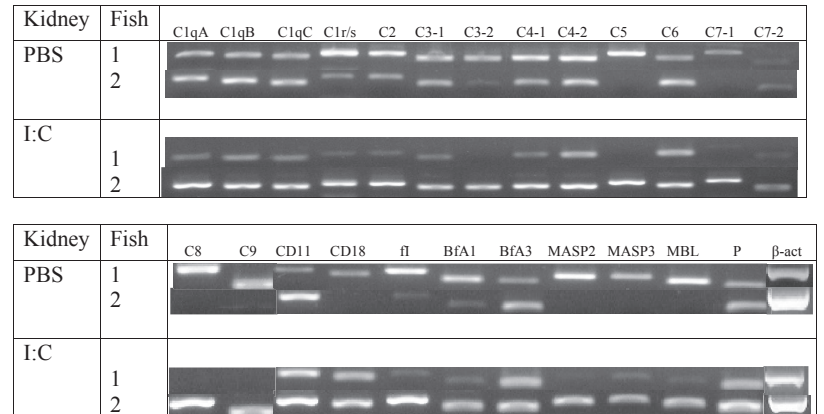

Fig. 3. Expression of the complement component genes in the kidneys. Abbreviations are shown in the Fig. 1 captions.

\section{Expression pattern in the gut}

The overall expression pattern was very similar to that of kidney, but the gut gave more steady expression in that most of the genes tested, showing signals in the two individuals tested both in control and stimulated groups (Fig. 4).
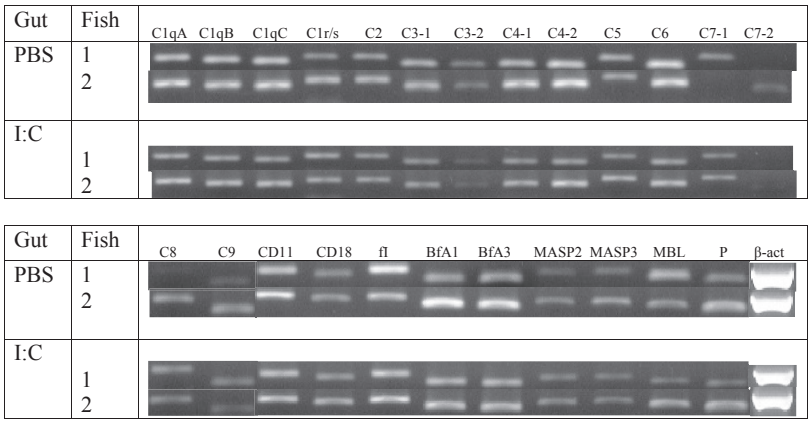

Fig. 4. Expression of the complement component genes in the guts. Abbreviations are shown in the Fig. 1 captions.

\section{Expression pattern in the hepatopancreas}

As shown in Fig. 5, all the transcripts were clearly detected in the two control fish, and the levels were almost unchanged in the stimulated fish, except for $\mathrm{C} 7-1$ and C7-2, of which signal was very faint or undetectable in the poly I:C-injected fish.

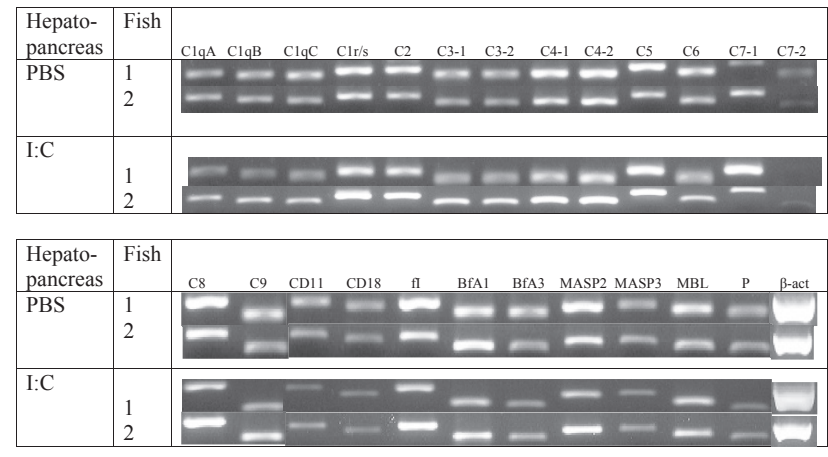

Fig. 5. Expression of the complement component genes in the hepatopancreas. Abbreviations are shown in the Fig. 1 captions.

\section{Expression pattern in the gonad}

As in gut and hepatopancreas, all the genes tested gave expression signals in the gonad of the control fish, and the expression levels showed no prominent up-regulation or down-regulation in the poly I:C-stimulated fish (Fig. 6).
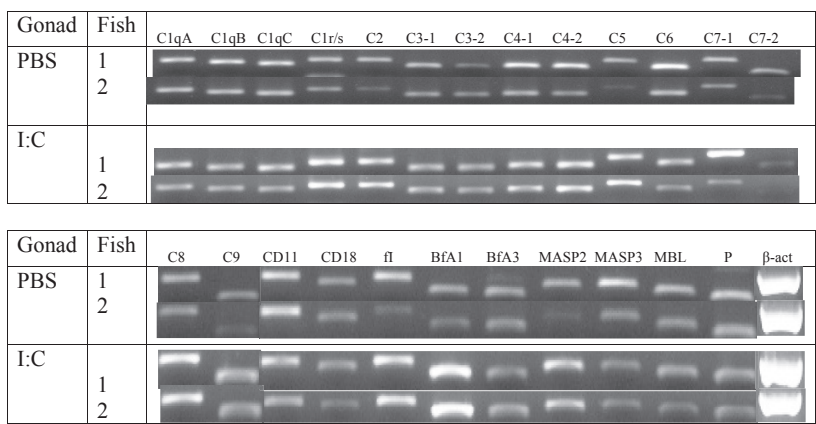

Fig. 6. Expression of the complement component genes in the gonads. Abbreviations are shown in the Fig. 1 captions. 


\section{Expression pattern in the heart}

The heart of the control fish showed rather limited expression of the complement components when compared with kidney and hepatopancreas, in that signals of $\mathrm{C} 5$, C8, and fI were almost missing, while B/C2-B, C3-2, $\mathrm{C} 7-1, \mathrm{C} 7-2, \mathrm{C} 9, \mathrm{~B} / \mathrm{C} 2-\mathrm{A} 1$, and MASP2 were observed only one of the two control fish (Fig. 7). In contrast, the poly I:C-stimulated fish showed more steady expression in both test fish, though the band intensities did not increase apparently.
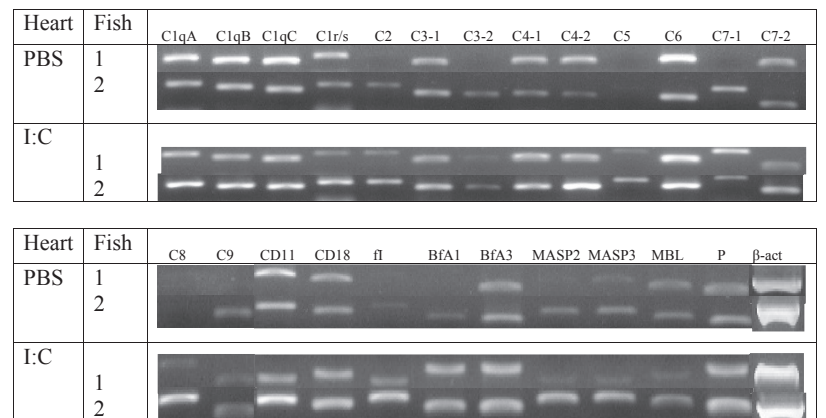

Fig. 7. Expression of the complement component genes in the hearts. Abbreviations are shown in the Fig. 1 captions.

\section{Expression pattern in the spleen}

In the control fish spleen, expressions of all the genes tested were detected from at least one of the two fish, showing substantial individual variations (Fig. 8). In the stimulated fish, however, both two test fish gave signals of expression, implying some up-regulating effect of poly $\mathrm{I}: \mathrm{C}$. In particular, band intensities of B/C2-B, C5, C7-1, $\mathrm{C} 8, \mathrm{~B} / \mathrm{C} 2-\mathrm{A} 1, \mathrm{MASP} 2$, and MBL became stronger in the stimulated fish than in the controls. In contrast, $\mathrm{C} 1 \mathrm{qA}, \mathrm{B}$, $\mathrm{C}$-chain expression levels become lower in the stimulated fish.

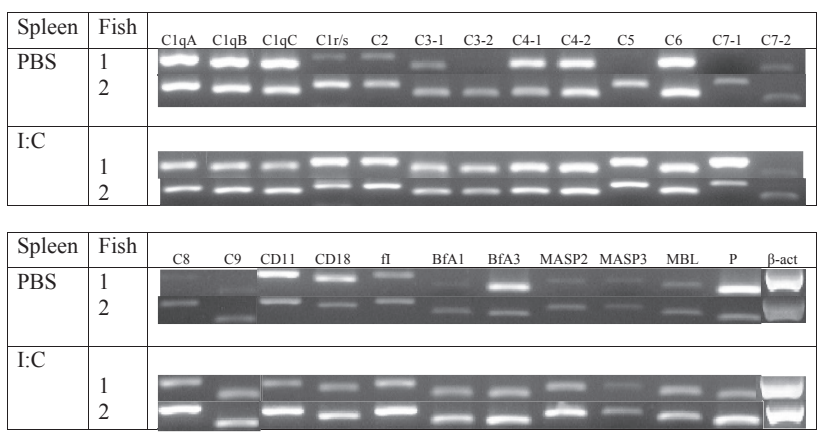

Fig. 8. Expression of the complement component genes in the spleens. Abbreviations are shown in the Fig. 1 captions.

\section{Expression pattern in the skin}

While transcripts of many complement genes were detected in the skin, signals of B/C2-B, C3-2, C5, C8, fI, $\mathrm{B} / \mathrm{C} 2-\mathrm{A} 1$, and MASP2 were faint or totally undetectable in the control fish (Fig. 9). In the stimulated fish, how-

Table 2. Tissue distribution of mRNAs encoding the complement components in normal zebrafish

\begin{tabular}{|c|c|c|c|c|c|c|c|c|c|}
\hline Gene & Gill & $\mathrm{Br}$ & $\mathrm{Kd}$ & Gut & Hp & $\mathrm{Gd}$ & $\mathrm{Ht}$ & $\mathrm{Sp}$ & Sk \\
\hline C1qA & + & + & ++ & ++ & ++ & ++ & ++ & ++ & + \\
\hline C1qB & + & + & ++ & ++ & ++ & ++ & ++ & ++ & + \\
\hline $\mathrm{C} 1 \mathrm{qC}$ & + & + & ++ & ++ & ++ & ++ & ++ & ++ & + \\
\hline $\mathrm{C} 1 \mathrm{r} / \mathrm{s}$ & + & + & ++ & + & ++ & + & + & ++ & + \\
\hline B/C2-B & \pm & - & + & + & ++ & + & \pm & + & - \\
\hline C3-1 & + & + & ++ & ++ & ++ & + & + & + & + \\
\hline C3-2 & \pm & - & + & + & ++ & + & \pm & \pm & \pm \\
\hline C4-1 & \pm & + & ++ & ++ & ++ & + & + & ++ & + \\
\hline C4-2 & + & + & ++ & ++ & ++ & + & + & ++ & + \\
\hline C5 & - & - & \pm & + & ++ & + & + & \pm & - \\
\hline C6 & + & + & + & ++ & ++ & ++ & ++ & ++ & + \\
\hline C7-1 & \pm & \pm & \pm & \pm & + & + & \pm & \pm & \pm \\
\hline $\mathrm{C} 7-2$ & + & + & + & \pm & + & + & + & + & \pm \\
\hline C8 & - & + & \pm & \pm & ++ & + & - & \pm & - \\
\hline C9 & \pm & \pm & \pm & + & ++ & + & \pm & + & \pm \\
\hline CD11 & ++ & ++ & ++ & ++ & ++ & ++ & ++ & ++ & + \\
\hline CD18 & + & \pm & \pm & + & ++ & + & + & + & + \\
\hline fI & \pm & + & + & ++ & ++ & + & \pm & + & \pm \\
\hline $\mathrm{B} / \mathrm{C} 2-\mathrm{A} 1$ & \pm & \pm & + & ++ & ++ & + & \pm & \pm & \pm \\
\hline $\mathrm{B} / \mathrm{C} 2-\mathrm{A} 3$ & + & + & + & + & ++ & ++ & + & + & + \\
\hline MASP2 & \pm & \pm & \pm & + & ++ & + & + & \pm & \pm \\
\hline MASP3 & \pm & \pm & \pm & + & ++ & ++ & + & + & \pm \\
\hline MBL & + & \pm & \pm & + & ++ & ++ & + & + & \pm \\
\hline $\mathrm{P}$ & + & + & + & ++ & ++ & ++ & + & + & + \\
\hline
\end{tabular}

*Relative expression levels compared among the organs in each gene are shown: + , weak or moderate expression; ++, strong expression; \pm , weak or moderate expression only in one of the two individuals tested. Abbreviations: Br, brain; Kd, kidney; Hp, hepatopancreas; Gd, gonad; Ht, heart; Sp, spleen; Sk, skin. 
ever, expression of these components became visible. In addition, the baseline expression of $\mathrm{C} 6, \mathrm{C} 7-1, \mathrm{C} 7-2$, CD11/CD18, MASP3, MBL, and P showed further increase. On the other hand, no significant change in the expression level was observed for C1qA, B, C-chains, C1r/s, C3-1, C4-1, C4-2, and B/C2-A3.
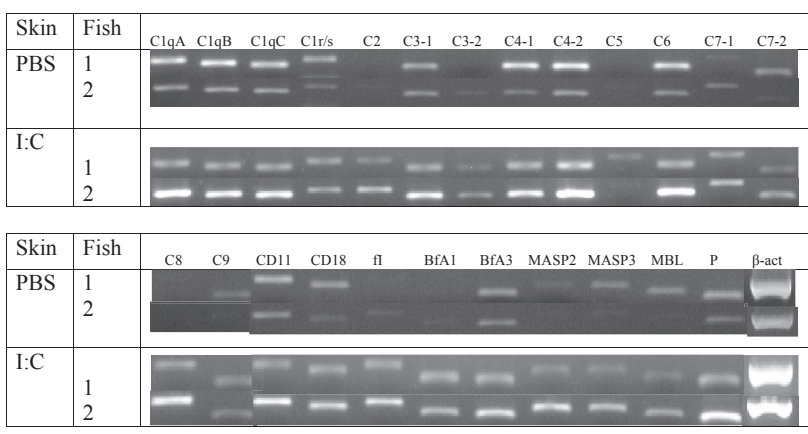

Fig. 9. Expression of the complement component genes in the skins. Abbreviations are shown in the Fig. 1 captions.

From the above results, normal tissue distribution of the complement components mRNA and their response to poly I:C-stimulation are summarized in Table 2 and Table 3, respectively.

\section{DISCUSSION}

Although the major site of complement component production in fish is considered to be liver or hepatopancreas as in mammals, it is increasingly probable that their extra hepatic expressions such as those in skin, spleen and kidney may have a functional significance of the complement system for first-line or local defense of fish (Lovoll et al., 2007a; Gonzalez et al., 2007ab). Thus more comprehensive findings on the tissue distribution of wider range of the complement components in fish.

Table II-2 summarizes the tissue distribution of transcripts from 24 genes encoding various complement components and their subunits based on the normal fish data in Figs. 1 to 9. As recognized in mammals, the hepatopancreas is a major production site for most components, but many of the components are synthesized in substantially wide range of organs. Among them, C1q, C1r/s, C3-1, C4-1, C4-2, C6 and properdin showed a broad tissue distribution of their mRNAs, suggesting that their basal extrahepatic expression would have significance in their local innate defense or in systemic complement synthesis, as inferred from the extrahepatic expression of C3, C4, C5, C7 and Bf in trout (Lovoll et al., 2007a).

It is reasonable that $\mathrm{C} 1 \mathrm{qA}, \mathrm{B}, \mathrm{C}$ subunits and $\mathrm{C} 1 \mathrm{r} / \mathrm{s}$ showed an essentially identical tissue distribution, as the three subunits form a $\mathrm{C} 1$ complex, the first complement component. Similarly, MBL, MASP2, and MASP3 showed almost identical tissue distribution. In contrast, distributions of CD11 and CD18 are considerably different from each other, despite that they should assemble an integrin molecule functioning as a complement receptor type 3 (CR3), specific for an activated fragment of C3 (Law and

Table 3. Summary of changes in the expression level of the complement components and their subunits in various organs of zebrafish after stimulation with poly I:C

\begin{tabular}{|c|c|c|c|c|c|c|c|c|c|}
\hline Gene & Gill & $\mathrm{Br}$ & $\mathrm{Kd}$ & Gut & Hp & $\mathrm{Gd}$ & $\mathrm{Ht}$ & $\mathrm{Sp}$ & Sk \\
\hline C1qA & $\uparrow$ & $\leftrightarrow$ & $\leftrightarrow$ & $\leftrightarrow$ & $\leftrightarrow$ & $\leftrightarrow$ & $\leftrightarrow$ & $\leftrightarrow$ & $\leftrightarrow$ \\
\hline $\mathrm{C} 1 \mathrm{qB}$ & $\uparrow$ & & $\leftrightarrow$ & $\leftrightarrow$ & $\leftrightarrow$ & $\leftrightarrow$ & $\leftrightarrow$ & $\leftrightarrow$ & $\leftrightarrow$ \\
\hline $\mathrm{C} 1 \mathrm{qC}$ & $\uparrow$ & $\leftrightarrow$ & $\leftrightarrow$ & $\leftrightarrow$ & $\leftrightarrow$ & $\leftrightarrow$ & $\leftrightarrow$ & $\leftrightarrow$ & $\leftrightarrow$ \\
\hline $\mathrm{C} 1 \mathrm{r} / \mathrm{s}$ & $\leftrightarrow$ & & $\leftrightarrow$ & $\leftrightarrow$ & $\leftrightarrow$ & $\uparrow$ & $\leftrightarrow$ & $\leftrightarrow$ & $\leftrightarrow$ \\
\hline $\mathrm{C} 2(\mathrm{~B} / \mathrm{C} 2-\mathrm{B})$ & $\uparrow$ & & $\leftrightarrow$ & $\leftrightarrow$ & $\leftrightarrow$ & $\uparrow$ & & $\leftrightarrow$ & $\uparrow$ \\
\hline C3-1 & $\uparrow$ & $\leftrightarrow$ & $\leftrightarrow$ & $\leftrightarrow$ & $\leftrightarrow$ & $\leftrightarrow$ & $\leftrightarrow$ & $\leftrightarrow$ & $\leftrightarrow$ \\
\hline C3-2 & $\uparrow$ & & & $\leftrightarrow$ & $\leftrightarrow$ & $\uparrow$ & & & $\uparrow$ \\
\hline C4-1 & $\uparrow$ & $\leftrightarrow$ & $\leftrightarrow$ & $\leftrightarrow$ & $\leftrightarrow$ & $\leftrightarrow$ & $\leftrightarrow$ & $\leftrightarrow$ & $\leftrightarrow$ \\
\hline C4-2 & $\uparrow$ & $\leftrightarrow$ & $\leftrightarrow$ & $\leftrightarrow$ & $\leftrightarrow$ & $\leftrightarrow$ & $\leftrightarrow$ & $\leftrightarrow$ & $\leftrightarrow$ \\
\hline C5 & & $\leftrightarrow$ & & $\leftrightarrow$ & $\leftrightarrow$ & & $\uparrow$ & & \\
\hline C6 & $\uparrow$ & & $\leftrightarrow$ & $\leftrightarrow$ & $\leftrightarrow$ & $\leftrightarrow$ & $\leftrightarrow$ & $\leftrightarrow$ & $\leftrightarrow$ \\
\hline C7-1 & $\uparrow$ & & & & $\leftrightarrow$ & $\leftrightarrow$ & & & \\
\hline C7-2 & $\uparrow$ & & & & & & $\leftrightarrow$ & $\leftrightarrow$ & \\
\hline C8 & & $\leftrightarrow$ & & & $\leftrightarrow$ & $\uparrow$ & $\uparrow$ & & $\uparrow$ \\
\hline C9 & & & & & $\leftrightarrow$ & $\uparrow$ & & $\uparrow$ & \\
\hline CD11 & $\leftrightarrow$ & $\leftrightarrow$ & $\leftrightarrow$ & $\leftrightarrow$ & $\downarrow$ & $\leftrightarrow$ & $\leftrightarrow$ & $\leftrightarrow$ & $\leftrightarrow$ \\
\hline CD18 & $\uparrow$ & & & $\leftrightarrow$ & $\downarrow$ & $\leftrightarrow$ & $\leftrightarrow$ & $\leftrightarrow$ & $\leftrightarrow$ \\
\hline fI & & $\leftrightarrow$ & & $\leftrightarrow$ & $\leftrightarrow$ & $\leftrightarrow$ & & $\leftrightarrow$ & $\uparrow$ \\
\hline $\mathrm{B} / \mathrm{C} 2-\mathrm{A} 1$ & $\uparrow$ & & $\leftrightarrow$ & $\leftrightarrow$ & $\leftrightarrow$ & $\uparrow$ & $\uparrow$ & $\uparrow$ & $\uparrow$ \\
\hline $\mathrm{B} / \mathrm{C} 2-\mathrm{A} 3$ & & & $\leftrightarrow$ & $\leftrightarrow$ & $\downarrow$ & $\leftrightarrow$ & $\leftrightarrow$ & $\leftrightarrow$ & $\leftrightarrow$ \\
\hline MASP2 & & & & $\leftrightarrow$ & $\leftrightarrow$ & & & $\uparrow$ & $\uparrow$ \\
\hline MASP3 & & & & $\leftrightarrow$ & $\downarrow$ & $\leftrightarrow$ & $\leftrightarrow$ & $\leftrightarrow$ & \\
\hline MBL & & & & $\leftrightarrow$ & $\leftrightarrow$ & $\leftrightarrow$ & $\leftrightarrow$ & $\uparrow$ & \\
\hline $\mathrm{P}$ & $\leftrightarrow$ & $\leftrightarrow$ & $\leftrightarrow$ & $\leftrightarrow$ & $\downarrow$ & $\leftrightarrow$ & $\leftrightarrow$ & $\leftrightarrow$ & $\leftrightarrow$ \\
\hline
\end{tabular}

Horizontal arrows indicate no significant change. Abbreviations: Br, brain; Kd, kidney; Hp, hepatopancreas; Gd, gonad; Ht, heart; Sp, spleen; Sk, skin. 
Reid 1995). The differential transcriptional level of CD11 and CD18 may have a unique regulation for the expression of functional CR3.

It should be noted that the gonad expresses all the complement genes tested. Although cellular localization of the transcripts and the proteins are still unknown, the presence of all the complement components in the gonad suggests their possible roles in reproduction and early development.

There are a number of reports regarding transcriptional response of the complement component genes, as well as other innate immune-relevant genes such as cytokines and the acute phase proteins, in several fish species after infection with bacteria, parasites or stimulation with vaccine or immunostimulants such as LPS and $\beta-1,3$-glucans.

In the rainbow trout, which has been best studied for complement expression responses, infection of a parasite (Icthyophthirius multifiliis) upregulated C3 expression in the skin and spleen and C5 and Bf expression in the spleen and head kidney, suggesting involvement of the complement system in anti-parasite defense mechanisms (von Gersdorff et al., 2008; Sigh et al., 2004). As for bacterial challenge, controversial results have been reported for trout; Yesinia ruckeri infection elicited down-regulation of C3, C5 and Bf expression in liver (Raida and Buchmann, 2009), whereas challenge with Aeromonas salmonicida and Flavobacterium psychrophilum did not affect C3 expression in liver, spleen, or head kidney (Overturf and LaPatra, 2006). Listonella anguillarum bacterin injected with Freunt's incomplete adjuvant suppressed C3 expression but elevated C7 level in trout liver (Gerwick et al., 2006). Furthermore, bacterial lip polysaccharides (LPS) and yeast $\beta$-glucans have been reported to modulate differentially expression levels of three C3 isotypes, C3-1, C3-3 and C3-4, in trout liver, spleen and head kidney, in which C3-1 was up-regulated but C3-4 was down-regulated (Lovoll et al., 2007b). As for response to viral infection, it is an only report that the infectious hematopoietic necrosis virus stimulated trout C3 expression (Overturf and LaPatra, 2006).

In carp, a similar expression response of C3 has been observed upon infection of a parasite, Trypanoplasma borreli (Saeij et al., 2003). LPS and $\beta$-glucan administrations have been reported to stimulate some complement components such as C5 and B/C2-A3, suggesting their ability to respond against bacterial and fungal infection (Gonzalez et al., 2007).

The present study showed responses of 24 complement components and their subunits after stimulation with poly I:C, a viral mimicry molecule. The most prominent expression response was observed in the gill, where transcriptions of C1, B/C2-B, C3, C4, C6, C7, and B/C2-A1 were up-regulated. Skin and gonad also showed elevated levels of several components as summarized in Table 3. In contrast, hepatopancreas, the major site of the basal expression, showed no change of the expression level or even down-regulation of a few components.

From the present results, it is considered that gill may play an important role in the innate immune response and defense against viral infection. Although the present experiment was performed using entire gill specimen containing blood cell in the vessels, the contribution of such contaminating blood cell to the expression profile of the gill is unlikely because other blood cell-rich organs like kidney, heart and spleen showed expression response pattern distinct from that of gill.

It is to be elucidated that how poly I:C modulated the complement expression in the gill. As poly I: $\mathrm{C}$ is a ligands for toll-like receptor 3 (TLR3) in mammals (Alexopoulou et al., 2001), and TLR3 has also been identified in various fish species including zebrafish (Jault et al., 2004), signaling through TLR3 and downstream cytokines such as IL-1 $\beta$ may have a role in the transcriptional enhancement of the complement expression in the gill. Elucidation of the functional significance of the up-regulated production of the complement components in the gill may provide an important clue to better understand anti-viral defense system in fish and contribute to development of countermeasure or prevention of viral diseases in aquaculture, in combination with other methods such as vaccination.

\section{ACKNOWLEDGEMENTS}

The authors thank Ms. Chie Okura for technical assistance. This study was supported in part by a grantin-aid from Japan Society for the Promotion of Science to $\mathrm{MN}$.

\section{REFERENCES}

Alexopoulou, L., A. C. Holt, R. Medzhitov, and R. A. Flavell 2001 Recognition of double-stranded RNA and activation of NF-kappaB by Toll-like receptor 3. Nature, 413: 732-738

Alvarez-Pellitero, P. 2008 Fish immunity and parasite infections: from innate immunity to immunoprophylactic prospects. Vet. Immunol. Immunopathol., 126: 171-198

Fortier, M. E., S. Kent, H. Ashdown, S. Poole, P. Boksa, G. N. Luheshi 2004 The viral mimic, polyinosinic:polycytidylic acid, induces fever in rats via an interleukin-1-dependent mechanism. Am. J. Physiol. Regul. Integr. Comp. Physiol. 287: 759-766

Gerwick, L., G. Corley-Smith, and C. J. Bayne 2007 Gene transcript changes in individual rainbow trout livers following an inflammatory stimulus. Fish Shellfish Immunol., 22: 157-171

Gonzalez, S. F., K. Buchmann, and M. E. Nielsen 2007a Complement expression in common carp (Cyprinus carpio L.) during infection with Ichthyophthirius multifiliis. Dev. Comp. Immunol., 31: 576-586

Gonzalez, S. F., N. Chatziandreou, M. E. Nielsen, W. Li, J. Rogers, R. Taylor, Y. Santos, and A. Cossins 2007b Cutaneous immune responses in the common carp detected using transcript analysis. Mol. Immunol, , 44: 1664-1679

Jault, C., L. Pichon, and J. Chluba 2004 Toll-like receptor gene family and TIR-domain adapters in Danio rerio. Mol. Immunol., 40: 759-771

Law, S. K. A. and K. B. M. Reid 1995 Complement, 2nd ed. by D. Male, IRL Press, Oxford (UK)

Løvoll, M., H. Johnsen, H. Boshra, J. Bøgwald, J. O. Sunyer, and R. A. Dalmo 2007a The ontogeny and extrahepatic expression of complement factor C3 in Atlantic salmon (Salmo salar). Fish Shellfish Immunol., 23: 542-552

Løvoll, M., U. Fischer, G. S. Mathisen, J. Bøgwald, M. Ototake, and R. A. Dalmo 2007b The C3 subtypes are differentially regulated 
after immunostimulation in rainbow trout, but head kidney macrophages do not contribute to C3 transcription. Vet. Immunol. Immunopathol., 117: 284-295

Nakao, M., T. Kajiya, Y. Sato, T. Somamoto, Y. Kato-Unoki, M. Matsushita, M. Nakata, T. Fujita, and T. Yano 2006 Lectin pathway of bony fish complement: identification of two homologs of the mannose-binding lectin associated with MASP2 in the common carp (Cyprinus carpio). J. Immunol., 177: $5471-5479$

Overturf, K. and S. LaPatra 2006 Quantitative expression of immunological factors in rainbow trout, Oncorhynchus mykiss, after infection with either Flavobacterium psychrophilum, Aeromonas salmonicida, or infectious haematopoietic necrosis virus. J. Fish Dis., 29: 215-224

Park, K. C., J. A. Osborne, S. C. Tsoi, L. L. Brown, and S. C. Johnson 2005 Expressed sequence tags analysis of Atlantic halibut (Hippoglossus hippoglossus) liver, kidney and spleen tissues following vaccination against Vibrio anguillarum and Aeromonas salmonicida. Fish Shellfish Immunol., 18: $393-415$

Peatman, E., P. Baoprasertkul, J. Terhune, P. Xu, S. Nandi, H. Kucuktas, P. Li, S. Wang, B. Somridhivej, R. Dunham, and Z. Liu 2007 Expression analysis of the acute phase response in channel catfish (Ictalurus punctatus) after infection with a Gram-negative bacterium. Dev. Comp. Immunol., 31: 1183-1196

Raida, M. K. and K. Buchmann 2009 Innate immune response in rainbow trout (Oncorhynchus mykiss) against primary and secondary infections with Yersinia ruckeri O1. Dev. Comp.
Immunol., 33: 35-45

Saeij, J. P., B. J. de Vries, and G. F. Wiegertjes 2003 The immune response of carp to Trypanoplasma borreli: kinetics of immune gene expression and polyclonal lymphocyte activation. Dev. Comp. Immunol, 27: 859-874

Selvaraj, V., K. Sampath, and V. Sekar 2005 Administration of yeast glucan enhances survival and some non-specific and specific immune parameters in carp (Cyprinus carpio) infected with Aeromonas hydrophila. Fish Shellfish Immunol., 19 293-306

Selvaraj, V., K. Sampath, and V. Sekar 2006 Adjuvant and immunostimulatory effects of beta-glucan administration in combination with lipopolysaccharide enhances survival and some immune parameters in carp challenged with Aeromonas hydrophila. Vet. Immunol. Immunopathol, 114: 15-24

Sigh, J., T. Lindenstrøm, and K. Buchmann 2004 The parasitic ciliate Ichthyophthirius multifilis induces expression of immune relevant genes in rainbow trout, Oncorhynchus mykiss. J. Fish Dis., 27: 409-417

Vo, K. T., M. Tsujikura, T. Somamoto, and M. Nakao 2009 Identification of cDNA sequences encoding the complement components of zebrafish (Danio rerio). J. Fac. Agr., Kyushu Univ., 54: in press

von Gersdorff Jorgensen, L., E. Nemli, R. D. Heinecke, M. K. Raida, and K. Buchmann 2008 Immune-relevant genes expressed in rainbow trout following immunisation with a live vaccine against Ichthyophthirius multifiliis. Dis. Aquat. Organ., 80 189-197 